\title{
Effet De La Fertilisation Phospho-Potassique Sur Le Rendement Grainier Et La Qualite Des Semences De Cajanus Cajan L. Millsp. Sur Un Ferrasol A Yamoussoukro, Region Centre De La Cote D'ivoire
}

\section{Faustin P. Koutouan, Ingénieur, Doctorant}

Institut National Polytechnique-Félix Houphouët Boigny de Yamoussoukro, Ecole doctorale, BP 1093, Yamoussoukro, Côte d'Ivoire

\section{Bodji C Nguessan, Ingénieur}

Institut National Polytechnique-Félix Houphouët Boigny de Yamoussoukro, Laboratoire de Zootechnie et Productions Animales, BP 1093,

Yamoussoukro, Côte d'Ivoire

Eboua N. Wandan, Ingénieur, PhD, Maitre de Conférences Institut National Polytechnique-Félix Houphouët Boigny de Yamoussoukro, Département Eaux et forêt et Environnement, BP 1313, Yamoussoukro, Côte d'Ivoire

\section{Djéhi B. Ta Bi, Ingénieur}

Ministère des Ressources Animales et Halieutiques (RCI), BP V 84 Abidjan, Côte d'Ivoire

\section{doi: 10.19044/esj.2017.v13n21p7 URL:http://dx.doi.org/10.19044/esj.2017.v13n21p7}

\begin{abstract}
The aim of the present work was to evaluate the effect of phosphorus and potassium fertilization on the production and the quality of pigeon pea seeds produced on a ferrasol type sol. A completely randomized block design was used, in a 2x3x3 factorial set with three replications. A density of 20000 plants $\mathrm{ha}^{-1}$ was used. The treatments were $0,40,80$, units for phosphorus $\left(\mathrm{P}_{2} \mathrm{O}_{5}\right)$ and potassium $(\mathrm{KCl})$. Treatment which received $\mathrm{P} 80 \mathrm{~K} 80$ fertilization had greater productivity $(650 \mathrm{~kg})$ but a low germination rate $(30 \%)$. The optimal dose $(523 \mathrm{~kg}$ and $90 \%$ germination rate) was obtained with P40K80, followed by $\mathrm{P} 40 \mathrm{~K} 40$ dose $(516 \mathrm{~kg}$ and $82 \%$ germination rate). Phosphorus and potassium had mutually acted and influenced positively the production and the quality of the seeds of $C$. cajan.
\end{abstract}

Keywords: Cajanus cajan, density, fertilization 


\section{Résume}

L'objectif de cette étude était d'évaluer l'influence de la fumure phospho-potassique sur le rendement grainier de Cajanus cajan (L.) (Fabacée). Il s'agissait d'évaluer la croissance en hauteur, le rendement grainier et le pouvoir germinatif des plants de Cajanus cajan sur le site de la station semencière de Toumbokro (Yamoussoukro). Chaque fertilisant a été testé à trois modalités $(0,40,80$ unités par hectare) à partir d'un dispositif expérimental constitué de trois blocs de Fisher en randomisation complète. Les résultats obtenus montrent que les hauteurs varient en fonction des traitements $(\mathrm{P}<0,05)$ et se situent entre 275 et $309 \mathrm{~cm}$ respectivement pour P0K0 et P80K80. Par ailleurs, on constate que les graines sont fortement attaquées par les insectes (notamment par Callosobruchus chinensis L., Coléoptère). Le rendement grainier varie en fonction des traitements et va de 0,24 à $0,65 \mathrm{t} / \mathrm{ha}$, respectivement pour P0K0 et P80K80. Quant aux taux de germination, les valeurs induites par les différents traitements se situent entre $30 \% \quad(\mathrm{P} 80 \mathrm{~K} 80)$ et $90 \% \quad(\mathrm{P} 40 \mathrm{~K} 80)$. Les différences observées sont significatives $(\mathrm{P}<0,05)$. Compte tenu des rendements enregistrés et de la qualité des semences testées, le traitement P40K80 semble être le plus indiqué pour assurer une bonne production grainière de Cajanus cajan.

Mots clés: Cajanus cajan, rendement, hauteur, fumure, germination

\section{Introduction}

Les sols de la savane africaine sont généralement déficients en éléments nutritifs, situation qui a des implications négatives sur l'intensification des cultures et de l'élevage et donc sur la sécurité alimentaire en Afrique (Odilon et al., 2007). Les longues périodes de jachère naturelle, utilisées autrefois pour régénérer la fertilité du sol, sont devenues obsolètes (Sedga, 1997; Odilon et al., 2007). De nouvelles alternatives sont développées, au travers de l'utilisation des légumineuses (Sedga, 199; Mando, 2007 ; Schulz et al., 2003; Aihou et al., 2006; Odilon et al., 2007); car ces espèces sont capables de fixer l'azote et de rétrocéder tout ou une partie au sol pour les cultures subséquentes (Messager, 1983; Mulongoy et Kang, 1986; Daniel et Ong, 1990; Bodji, 1992; Odilon et al., 2007), d'étouffer efficacement les adventices (Skerman et al., 1982; Odilon et al., 2007) et de limiter l'érosion, permettant ainsi d'améliorer les rendements agricoles (Dahiya et al., 2002; Odilon et al., 2007).

Cajanus cajan L. Millsp., est une légumineuse arbustive restauratrice des sols dégradés (Daniel et Ong, 1990; Agyare et al., 2002; Bodji et al., 2006; Aihou et al., 2006; Ogbe et Bamidele, 2007) et stabilisatrice des terrasses de conservation du sol (Kimani, 2000). Utilisée en association avec 
les autres cultures, elle permet de limiter la compétition pour la lumière, l'eau et les nutriments (Mergeai et $a l ., 2000$; Snapp et al., 2002; Snapp et al., 2003). Elle a un effet nettoyant et permet de lutter efficacement contre les mauvaises herbes (Ndabalishé, 1995; Schulz et al., 2003; Snapp et al., 2002). En rotation avec les céréales, elle permet d'améliorer le rendement de ces dernières (Dahiya et $a l .$, 2002). De plus, elle est très bien appétée par les petits ruminants (Wallis et al., 1986) et peut leur fournir un aliment de haute qualité qui pourrait être utilisé comme fourrage de base ou supplément alimentaire (Rao et $a l$., 2000 ; Shenkute et $a l ., 2013$ ). Elle s'intègre bien dans les systèmes de production culture-bétail (Agyare et al., 2002; Defly, 2004).

En Côte d'Ivoire, elle était intégrée initialement dans les systèmes d'exploitation agricoles des populations du nord-est, qui en consomment les grains comme du haricot (Ndabalishe, 1995).

Cependant, certains résultats de la recherche ont changé la donne. Il s'agit entre autres, des essais qui ont prouvé que la rotation riz avec $C$. cajan améliore significativement le rendement du riz (Gbakatchétché et al., 2010) et ceux qui ont montré que cette plante pouvait être utilisée en zone cotonnière, dans les jachères améliorées de courte durée ( 3 ans) et que la remise en culture induisait un meilleur rendement du coton-grain comparativement à la culture cotonnière réalisée dans les conditions édictées par la Compagnie Ivoirienne du Développement du textile (CIDT) (Bodji et al., 2006). Dès lors, et au vu des précédents résultats dans le monde énumérés ci-dessus, il est apparu sur le territoire ivoirien un regain d'intérêt pour C. cajan comme plante à intégrer dans les systèmes AgricultureElevage. Il s'agit pour les agro-éleveurs de l'utiliser pour améliorer les jachères, supplémenter leur bétail et récolter le maximum de graines pour d'une part leur auto-consommation et d'autre part pour l'extension des superficies des jachères améliorées. Il se pose du coup un problème de disponibilité de semences de qualité. L'utilisation optimale d'engrais chimiques pourrait permettre de résoudre cet épineux problème, car la fertilisation de $C$. cajan entraine un bon développement végétatif et une augmentation du rendement grainier (Ahlawat et Saraf, 1981; Tayo et Togun, 1984).

Les données existantes sur la fertilisation de $C$. cajan montrent que la réponse de cette espèce à la fertilisation dépend de la variété, de la classe du sol, de son $\mathrm{pH}$, du statut de fertilité, du type de fertilisant (ternaire, binaire, fumure organique, etc.) du système de culture (intercalaire ou pure), du calendrier de fertilisation et du mode d'épandage et de gestion de la fertilisation (Tayo et Togun, 1984; Adu-Gyamfi et al., 1997a; Adu-Gyamfi et al., 1997b; Katayama et al., 1999; Owino-Gerroh et al., 2005; Blaise et al., 2005; Bertolin et al., 2008; Kimaro et al., 2009 ; Fageria et al., 2013 ; Fageria et Baligar, 2014 ; Babu et al., 2014). Malheureusement, il n'existe 
pas de données relatives à la fertilisation d'une culture de $C$. cajan sur un ferrasol en zone subhumide.

La présente étude a pour objectif de déterminer la dose optimale d'engrais binaire (K-P) économiquement rentable dans le cadre d'une amélioration du rendement grainier et de la qualité des semences de $C$. cajan sur un ferrasol en zone subhumide de la Côte d'Ivoire.

\section{Matériel et Méthodes}

\section{Zone d'étude}

L'étude a été conduite dans la région centre de la Côte d'Ivoire, précisément dans le District Autonome de Yamoussoukro (Figure 1). Ce district est situé dans la zone $30 \mathrm{~N}$ entre $725000 \mathrm{~m}$ et $785000 \mathrm{~m}$ de latitude Nord et $220000 \mathrm{~m}$ et $285000 \mathrm{~m}$ de longitude Ouest, à $248 \mathrm{~km}$ au nord d'Abidjan, la capitale économique (ANADER, 2006). La flore est dominée par la savane pré-forestière parsemée plus ou moins densément de petits arbres. On y trouve également des bosquets, des îlots forestiers et des galeries forestières qui se rencontrent dans les bas-fonds, le long des marigots et des fleuves. De nombreuses espèces fourragères appartenant aux genres Panicum, Stylosanthes, Andropogon, etc. colonisent également le district de Yamoussoukro (ANADER, 2006). Le climat de type équatorial, est caractérisé par quatre saisons. La grande saison des pluies se déroule de mi-mars à mi-juillet, la petite de mi-septembre à mi-novembre. La petite saison sèche va de mi-juillet à mi-septembre tandis que la grande a lieu de mi-novembre à mi-mars, avec la présence de l'harmattan. La pluviométrie moyenne se situe entre 900 et $1100 \mathrm{~mm}$ par an, avec une répartition spatiale très variable dans l'année et d'une année à l'autre. La température moyenne de la région est d'environ $26{ }^{\circ} \mathrm{C}$ (ANADER, 2006). L'expérience s'est déroulée à la Station Semencière de Yamoussoukro (SSY), sur un ferrasol de pH 6,5. La pluviométrie annuelle moyenne enregistrée de 2005 à 2015 sur cette station est de $1162 \mathrm{~mm}$. Le sol est à dominance sable $(77,25 \%)$ et limon $(8,25 \%)$ avec $1,7 \%$ de carbone organique. 


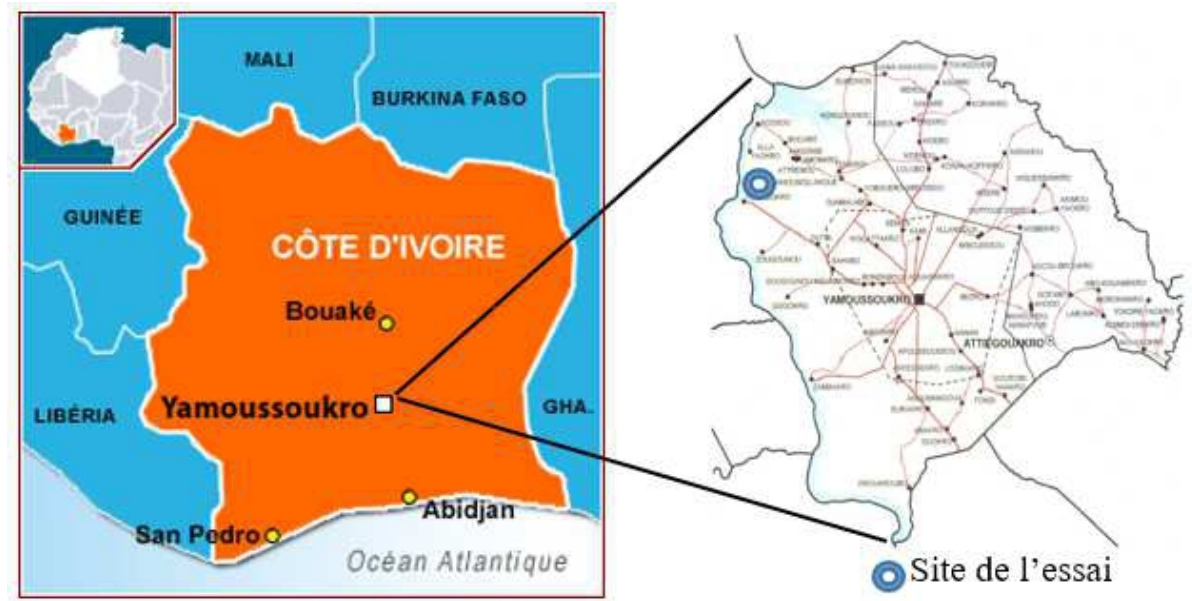

Figure 1. Carte de Côte d'Ivoire montrant le District Autonome de Yamoussoukro

\section{Matériel végétal}

La variété de $C$. cajan utilisée est une variété locale à cycle intermédiaire (150 à 200 jours). Ses semences sont de couleur rouge et ont été achetées sur le marché local de la ville de Yamoussoukro.

\section{Méthodes}

Le semis a été réalisé en poquets. La densité de semis adoptée est celle relative à la jachère améliorée à base de $C$. cajan, c'est-à-dire de 20000 plants/ha (1 m x 0,50 m) (Skerman et al., 1982). Deux sarclages ont été effectués les 15ème et 35ème jours après le semis (JAS). Les apports de fertilisants ont été effectués 36 JAS. L'engrais a été épandu de façon localisée autour des pieds de $C$. cajan. Un insecticide (Furadan) et un fongicide (Ivory 80) ont été utilisés pour le contrôle des ennemis et maladies. Le démariage a été réalisé au $30^{\mathrm{e}} \mathrm{JAS}$. L'engrais binaire ( $\mathrm{P}$ et $\mathrm{K}$ ) a été utilisé, car l'azote à la mise en place de $C$. cajan n'est pas forcément nécessaire (Kimaro et al., 2009).

\section{Dispositif expérimental}

Le site a été subdivisé en 3 blocs et chaque bloc comportait 9 parcelles élémentaires de $12 \mathrm{~m}^{2}(4 \mathrm{~m} \times 3 \mathrm{~m})$. Le dispositif comportait 9 traitements avec 3 répétitions, correspondant à différents niveaux de combinaison de phosphore et de potassium (tableau 2).

Le phosphore était sous forme de phosphate tricalcique $\left(\mathrm{Ca}_{3}\left(\mathrm{PO}_{4}\right)_{2}\right)$ avec $34 \%$ de $\mathrm{P}_{2} \mathrm{O}_{5}$ et le potassium était sous forme de chlorure de potassium $(\mathrm{KCl})$ avec $60 \%$ de $\mathrm{K}_{2} \mathrm{O}$. Chaque traitement a été reparti de façon aléatoire dans chacun des 3 blocs. 
Tableau 1. Traitements testés

\begin{tabular}{ccccc}
\hline \multirow{2}{*}{ Traitement } & \multicolumn{2}{c}{ Unités fertilisantes } & \multicolumn{2}{c}{ Equivalent en $\mathrm{Kg} / \mathrm{ha}$} \\
\cline { 2 - 5 } & $\mathrm{P}$ & $\mathrm{K}$ & $\mathrm{P}_{2} \mathrm{O}_{5}$ & $\mathrm{KCl}$ \\
\hline \hline T1 (témoin) & 0 & 0 & 0 & 0 \\
T2 & 0 & 40 & 0 & 56 \\
T3 & 0 & 80 & 0 & 112 \\
T4 & 40 & 0 & 98 & 0 \\
T5 & 40 & 40 & 98 & 56 \\
T6 & 40 & 80 & 98 & 112 \\
T7 & 80 & 0 & 196 & 0 \\
T8 & 80 & 40 & 196 & 56 \\
T9 & 80 & 80 & 196 & 112 \\
\hline
\end{tabular}

\section{Variables mesurées}

Les variables mesurées sont le rendement grainier et le taux de germination.

Pour le rendement grainier, l'unité de mesure était constituée de 12 plants préalablement identifiés dans les 2 lignes centrales. Les plants de bordure n'ont pas été concernés par les choix. Les gousses ont été récoltées manuellement 4 mois après semis et 5 fois au cours du cycle. Pour chaque récolte, les semences du même traitement sont combinées et séchées pendant 2-3 jours à l'air ambiant. Les graines ont été ensuite rassemblées et pesées, puis le rendement annuel par traitement a été calculé à l'aide de la formule suivante.

Rendement $\left(\frac{\mathrm{Kg}}{\mathrm{ha}} / \mathrm{an}\right)=\frac{Q}{12 \text { pieds }} \times 20000$ pieds où $\mathrm{Q}$ est la somme des productions des sous parcelles par traitement et 12 l'unité de mesure.

Concernant le taux de germination, pour chaque lot de semences, 150 graines à raison de 50 graines par boîte de pétri ont servi au test de germination. Les substrats utilisés sont du papier buvard et du coton hydrophile.

\section{Analyse des données}

Les données recueillies ont été soumises à une analyse de variance ANOVA à 95\% d'intervalle de confiance à l'aide du logiciel STATISTICA 7.1. La comparaison des moyennes a été faite selon le test de DUNCAN.

\section{Résultats et Discussion Résultats \\ Rendements grainiers}

Les rendements grainiers des différents traitements sont consignés dans le tableau 3. Le traitement témoin (T0) a enregistré le faible rendement (240 kg/ha) tandis que le traitement T9 (P80K80) a obtenu le rendement le 
plus élevé $(650 \mathrm{~kg} / \mathrm{ha})$. Cependant, les rendements des traitements $\mathrm{T} 3$ (P0K80), T6 (P40K80) et T8 (P80K40)) ne diffèrent pas significativement de celui de T9 (P80K80.

Tableau 2. Rendements grainiers des différents traitements

\begin{tabular}{cc}
\hline Traitements & Rendements (Kg/ha) \\
\hline T1 (P0K0) & $240 \pm 0,000 \mathrm{~d}$ \\
T2 (P0K40) & $398 \pm 84 \mathrm{c}$ \\
T3 (P0K80) & $600 \pm 14 \mathrm{a}$ \\
T4 (P40K0) & $425 \pm 100 \mathrm{c}$ \\
T5 (P40K40) & $516 \pm 43 \mathrm{~b}$ \\
T6 (P40K80) & $523 \pm 23 \mathrm{a}$ \\
T7 (P80K0) & $381 \pm 1 \mathrm{c}$ \\
T8 (P80K40) & $599 \pm 16 \mathrm{a}$ \\
T9 (P80K80) & $650 \pm 5 \mathrm{a}$ \\
\hline
\end{tabular}

NB : Dans la colonne, les chiffres portant les mêmes lettres ne diffèrent pas significativement $(p>0,05)$

\section{Pouvoir germinatif}

Comme le montre le tableau 4, les taux de germination varient de $30 \pm 6,0$ à $90 \pm 7,9 \%$. T9 (P80K80) a présenté le taux de germination le plus faible (30\%) tandis que T6 (P40K80) a enregistré le taux le plus élevé (90\%). Cependant, il n'existe pas de différence significative entre les résultats des tests de germination de T6 (P40K80) et T5 (P40K40). Par ailleurs, aucune différence significative n'existe non plus entre les taux de germination des traitements T1 (P0K0), T2 (P0K40), T3 (P0K80) et T7 (P80K0) et celui de T5 (P40K40).

Tableau 3. Résultats des tests de germination

\begin{tabular}{cc}
\hline Traitements & Taux de germination (\%) \\
\hline T1 (P0K0) & $64 \pm 15,00 \mathrm{bc} *$ \\
T2 (P0K40) & $62 \pm 16,82 \mathrm{bc}$ \\
T3 (P0K80) & $66 \pm 12,17 \mathrm{bc}$ \\
T4 (P40K0) & $60 \pm 15,40 \mathrm{c}$ \\
T5 (P40K40) & $82 \pm 4,00 \mathrm{ab}$ \\
T6 (P40K80) & $90 \pm 7,94 \mathrm{a}$ \\
T7 (P80K0) & $64 \pm 0,00 \mathrm{bc}$ \\
T8 (P80K40) & $60 \pm 3,79 \mathrm{c}$ \\
T9 (P80K80) & $30 \pm 6,00 \mathrm{~d}$ \\
\hline
\end{tabular}

* Dans la colonne, les chiffres portant les mêmes lettres ne diffèrent pas significativement $(\mathrm{p}>0,05)$

\section{Discussion}

En l'absence du phosphore, le rendement grainier croît à mesure que la dose de potassium augmente. A l'inverse, en l'absence de potassium, le 
rendement croît de $\mathrm{P} 0$ à $\mathrm{P} 40$ puis régresse de $\mathrm{P} 40(425 \mathrm{~kg})$ à $\mathrm{P} 80(310 \mathrm{~kg})$ même si la différence entre ces 2 valeurs est non significative. Pour le potassium donc, jusqu'à 80 unités soit $112 \mathrm{~kg} / \mathrm{ha}$ de $\mathrm{K} 2 \mathrm{O}$, il y'a une réponse. Ces résultats sont en accord avec l'affirmation de Klein et al. (2014) selon laquelle une dose importante de potassium permet un bon rendement en graine. Cependant, pour le phosphore, l'optimum se situerait entre 40 et 80 unités, c'est-à-dire entre $98 \mathrm{~kg} / \mathrm{ha}$ et $196 \mathrm{~kg} / \mathrm{ha}$ de P2O5. A 80 unités de phosphore, il y'a sans doute une interaction négative entre le $\mathrm{P}$ avec les paramètres symbiotiques comme l'ont signalé Aihou et al. (2006). Chez la variété améliorée à cycle court cv. IAPAR-43 Arata, Bertolin et al. (2008) ont observé au Brésil que le rendement diminue à mesure que la dose de potassium $\left(\mathrm{K}_{2} \mathrm{O}\right)$ augmentait. Il est passé de $2776 \mathrm{~kg} / \mathrm{ha}$ à la dose 0 à 2638 $\mathrm{kg} / \mathrm{ha}$ à la dose $30 \mathrm{~kg} / \mathrm{ha}$ puis à $2573 \mathrm{~kg} / \mathrm{ha}$ à la dose $90 \mathrm{~kg} / \mathrm{ha}$. Concernant le phosphore $\left(\mathrm{P}_{2} \mathrm{O}_{5}\right)$, les résultats montrent que les rendements passent de 2558 $\mathrm{kg} / \mathrm{ha}$ à la dose 0 à $2828 \mathrm{~kg} / \mathrm{ha}$ à la dose $160 \mathrm{~kg} / \mathrm{ha}$ de $\mathrm{P} 2 \mathrm{O} 5$. Ces différents résultats, parfois contradictoires pourraient suggérer qu'en ce qui concerne la fertilisation de C. cajan, chaque variété constitue un cas particulier. En effet, selon les analyses des résultats de plusieurs auteurs (Tayo et Togun, 1984; Adu-Gyamfi et al., 1997a; Adu-Gyamfi et al., 1997b; Katayama et al., 1999; Owino-Gerroh et al., 2005; Bertolin et al., 2008; Kimaro et al., 2009 ; Fageria et al., 2013 ; Fageria et Baligar, 2014), la réponse de C. cajan à la fertilisation dépend de la classe du sol, de son $\mathrm{pH}$, du statut de fertilité (riche en azote, en phosphore et en potassium ou non, fumure ou non), du type de fertilisant (ternaire, binaire, fumure organique, etc.) du système de culture (intercalaire ou pure), du calendrier de fertilisation, du mode d'épandage et de gestion de la fertilisation et de la variété.

Par ailleurs, l'analyse des rendements montre qu'il y a une interaction entre $\mathrm{P}$ et $\mathrm{K}$. Le meilleur rendement $(650 \mathrm{~kg})$ a été obtenu avec la dose P80K80. Ce résultat nous indique que le potassium régule les effets néfastes d'une dose élevée en phosphore. En effet, en l'absence de potassium, le rendement de $\mathrm{P} 80 \mathrm{~K} 0$ a chuté. Il est passé de $250 \mathrm{~kg} / \mathrm{ha}(\mathrm{P} 0 \mathrm{~K} 0)$ à $425 \mathrm{~kg}$ $(\mathrm{P} 40 \mathrm{~K} 0)$ puis à $310 \mathrm{~kg}(\mathrm{P} 80 \mathrm{~K} 0)$. La dose $\mathrm{P} 80 \mathrm{~K} 80$, certes présente le meilleur rendement mais reviendrait chère comparativement aux doses P0K80 (600 $\mathrm{kg})$ et $\mathrm{P} 40 \mathrm{~K} 80(523 \mathrm{~kg})$ qui enregistrent statistiquement les mêmes rendements que P80K80 mais qui ont l'avantage d'être moins couteux. En outre, le rendement de P40K40 (516 kg) est également proche de ceux des 3 doses précédentes même s'ils différent significativement.

En mettant en rapport les rendements et les taux de germination, la dose P40K80 (523 kg et 90\% de germination) est la dose optimale. Après elle, vient la dose P40K40 (516 kg et 82\% de germination). En effet, le taux de germination de qualité des semences de $C$. cajan défini par la FAO (2007) est de $70 \%$ au minimum. 
Le rendement du lot témoin est en conformité avec ceux observés par Velay et al. (2001) pour lesquels les variétés locales ont un rendement inférieur ou légèrement supérieur à $300 \mathrm{~kg}$. Les rendements très supérieurs à $300 \mathrm{~kg}$ obtenus dans cette étude sont selon toute vraisemblance dus à la fertilisation. Ils seraient certainement plus importants si une densité plus faible avait été adoptée. En effet, le rendement maximal en semence par variété est atteint avec des densités relativement faibles (Akinola et whiteman, 1974; Skerman et al., 1982). Skerman et al., (1982) avancent pour

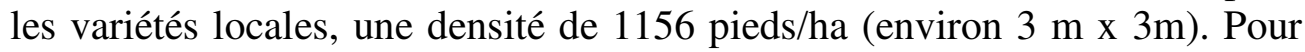
Akinola et whiteman (1974), à des densités plus élevées, il y a une augmentation de la mortalité des plants et un nombre réduit de gousses par plante; ce qui entraîne de graves réductions des rendements grainiers. Cependant, les travaux de Bertolin et al. (2008) ont montré le contraire. Selon ces auteurs, lorsque les plants sont serrés, ils produisent plus de graines qui germent moins. Mais lorsqu'ils sont séparés les uns des autres, ils produisent peu relativement au cas précédent mais germent plus. Dans tous les cas, Skerman et al. (1982) et Bertolin et al. (2008) sont d'accord à dire qu'il faut une densité faible pour avoir de bons taux de germination.

Concernant cet aspect, en l'absence de phosphore, le potassium n'influence pas le taux de germination quelle qu'en soit la dose. De même, en l'absence de potassium, le taux de germination reste quasiment le même quelle que soit la dose en phosphore. Bertolin et al. (2008) ont observé le contraire. Ils ont constaté sur une variété améliorée à cycle court que le phosphore à lui seul affectait positivement la qualité des semences. Toutefois, le phosphore et le potassium ont interagi sur le taux de germination. Ce qui confirme les propos de Klein et al. (2014) selon lesquels chez les légumineuses, le phosphore et la potasse jouent un rôle important pour la floraison, la grenaison et le remplissage des graines en cours de végétation et en fin de cycle. A la forte dose de phosphore (P80), le rendement croît avec le niveau de potassium. A l'inverse, le taux de germination baisse. Cela laisse penser qu'à une dose élevée d'engrais binaire (P80K80), le phosphore et le potassium impactent négativement la physiologie germinative des graines.

\section{Conclusion}

$\mathrm{Au}$ terme de cette étude, nous constatons que le potassium et le phosphore sont à la fois indispensable à une bonne production des graines et à une bonne qualité de ces dernières. Mais leur apport doit être raisonné afin d'éviter les effets pervers d'une trop forte dose. Pour les variétés locales ivoiriennes et, sur ferrasol, les doses semblent être comprises entre P40K40 et P40K80. A la densité adoptée, les paysans ont non seulement une couverture totale de leur parcelle, une limitation de leur enherbement, une 
accélération de la restauration mais en plus une production suffisante en graines de meilleure qualité pour assurer leur sécurité alimentaire et procéder à l'extension des jachères améliorées.

Cependant, la reprise d'une telle étude sur deux ou trois sites au moins et pendant trois ou quatre années conforterait ces résultats. Aussi, une étude économique comparative des effets de jachère améliorée de Cajanus cajan et de jachère naturelle sur le rendement des cultures subséquentes (coton ou maïs) devra-t-elle être menée pour mieux apprécier l'intérêt d'une fertilisation phospho-potassique dans une jachère améliorée à base de $C$ cajan.

\section{References:}

1. Adu-Gyamfi J. J., Ito, O., Yoneyama, T., Katayama K., 1997a. Nitrogen management and biological nitrogen fixation in sorghum/pigeonpea intercropping on Alfisols of the semi-arid tropics. Soil science and plant nutrition, Volume 43, SI, 1061-1066

2. Adu-Gyamfi J. J., Ito, O., Yoneyama, T., Devi G., Katayama K., 1997b. Timing of $\mathrm{N}$ fertilization on $\mathrm{N} 2$ fixation, $\mathrm{N}$ recovery and soil profile nitrate dynamics on sorghum/pigeonpea intercrops on Alfisols on the semi-arid tropics. Nutrient Cycling in Agroecosystems 48: 197-208, 1997.

3. Agyare W. A., Kombiok J. M., Karbo N., Larbi A., 2002. Management of pigeon pea in short fallows for crop-livestock production systems in the Guinea savanna zone of northern Ghana. Agroforestry Systems 54: 197-202

4. Ahlawat I. P. S., Saraf C. S., 1981. Response of pigeon pea (Cajanus cajan (L.) Millsp.) to plant density and phosphorus fertilizer under dryland conditions. J. agric. Sci., Camb., 97, 119-124

5. Aihou K., Sanginga N., Vanlauwe B., Diels J., Merckx R.; Van Cleemput O., 2006. Soil factors limiting growth and establishment of pigeon pea (Cajanus cajan (L.) Millsp. in farmers' fields in the derived savannah of the Benin. Bulletin de la Recherche Agronomique du Bénin, n52, pp 12-21

6. Akinola J. O., whiteman P. C., 1974. Agronomic Studies on Pigeon Pea (Cajanus cajan (L.) Millsp.). II. Responses to Sowing Density. Aust. J. Agric. Res., 26, 57-66

7. ANADER 2006. Monographie du Département de Yamoussoukro. Côte d'Ivoire, $55 \mathrm{p}$.

8. Babu S., Rana D. S., Yadav G. S., Singh R., 2014. Growth, yield, quality and nutrient content of pigeonpea (Cajanus cajan) as influenced by sunflower stover and nutrients management under pigeonpea-sunflower (Helianthus annuus) cropping system. 
9. Bertolin D. C., Sá M. E., Buzetti S., Santiago D., Barbosa R. M., 2008. Produção e qualidade de sementes de guandu: efeitos de doses de fósforo, potássio e espaçamentos em duas épocas de semeadura. Maringá, v. 30, n. 3, p. 415-419

10. Blaise D., Majumdar G., Tekale K.U., 2005. On-farm evaluation of fertilizer application and conservation tillage on productivity of cotton + pigeonpea strip intercropping on rainfed Vertisols of central India. Soil \& Tillage Research 84, 108-117

11. Bodji N. C., 1992. Arrière-effet de deux années de culture de légumineuses fourragères tropicales sur une culture de maïs. Communication au Symposium sur le Stylosanthes à kaduna-Nigéria du 24 au 31 octobre 1992, 23 p.

12. Bodji N. C., Ouattara Z., Koné S., Yapi Y. M., N'guessan. A. A., N'da K. N., Sebe P., Ouattara B., 2006. Intégration des cultures fourragères dans les systèmes de production de Bouafla (Bouaflé), en Côte d'Ivoire. Rapport final projet conjoint ESA-CIDT-ILRICORAF-IDRC, 60 pages+annexes

13. Dahiya S. S., Chauhan Y. S., Johansen C. , Waldia R. S., Sekhon H. S., Nandal J. K., 2002. Extra-short-duration pigeonpea for diversifying wheat-based cropping systems in the sub-tropics. Expl Agric., volume 38, pp. 1-11

14. Daniel N. J., Ong K. C., 1990. Perennial pigeonpea: a multi-purpose species for agroforestry systems. Agroforestry Systems 10: 113-129

15. Defly A., 2004. Intégration des cultures fourragères dans les systemes agropastoraux des zones à fortes pressions démographiques du sudest du Togo. In Dicko M., Ehouinsou M., Aboh A.B., Desquesnes M. (Eds): "Introduction des plantes fourragères dans les systèmes de production en Afrique de l'Ouest". CIRDES, PROCORDEL, actes de l'atelier tenu à Cotonou (Bénin), du 19 au 21 janvier 2004, pp 45-53

16. Fageria N. K., Ferreira E. P. B., Baligar V. C., Knupp A. M., 2013. Growth of Tropical Legume Cover Crops as Influenced by Nitrogen Fertilization and Rhizobia. Communications in Soil Science and Plant Analysis, 44:21, 3103-3119, DOI: 10.1080/00103624.2013.832283

17. Fageria N. K., Baligar V. C., 2014. Macronutrient-Use Efficiency and Changes in Chemical Properties of an Oxisol as Influenced by Phosphorus Fertilization and Tropical Cover Crops, Communications in Soil Science and Plant Analysis, 45:9, 1227-1246, DOI: 10.1080/00103624.2013.874030

18. FAO, 2007. Système des semences de qualité déclarée. Etude FAO Production Végétale et Protection des Plantes 185, 264 p. 
19. Gbakatchétché H; Sanogo S.; Camara M.; Bouet A., Kéli Z. J., 2010. Effet du paillage par des résidus de pois d'angole (Cajanus cajan L.) sur le rendement du riz (Oryza sativa) pluvial en zone forestière de Côte d'Ivoire. Agronomie Africaine 22 (2) : 131 - 137

20. Katayama K., Ito O., Adu-Gyamfi , J. J., Rao T. P., Dacanay E. V., Yoneyama T., 1999. Effects of NPK fertilizer combinations on yield and nitrogen balance in sorghum or pigeonpea on a vertisol in the semi-arid tropics, Soil Science and Plant Nutrition, 45:1, 143-150, DOI:10.1080/00380768.1999.10409330

21. Kimani M. P., 2000. Pigeonpea Breeding: Objectives, Experiences, and Strategies for Eastern Africa. In Silim N. S., Mergeai G., Kimani M. P. (Eds): " Status and Potential of Pigeonpea in Eastern and Southern Africa". Proceedings of a Regional Workshop Nairobi, Kenya, 12-15 Sep 2000, pp 21-32

22. Kimaro A. A., Timmer V.R., Chamshama S.A.O., Ngaga Y.N., Kimaro D.A., 2009. Competition between maize and pigeonpea in semi-arid Tanzania: Effect on yields and nutrition of crops. Agriculture, Ecosystems and Environment 134, 115-125

23. Klein H.-D., Rippstein G., Huguenin J., Toutain B., Guerin H., Louppé D., 2014. Les cultures fourragères. Quae, CTA, les presses agronomiques de Gembloux, $262 \mathrm{p}$

24. Mando A., 2007. Introduction à la Gestion Intégrée de la Fertilité des Sols (GIFS) : Principes des aspects technologiques. Notes présentées à la formation Internationale sur la Gestion Intégrée de la Fertilité des Sols (GIFS), Fada, 29 mai au 1er juin 2007, IFDC, 35 p

25. Mergeai G., Silim N. S., Baudoin J. P., 2000. Improved Management Practices to Increase Productivity of Traditional Cereal/Pigeonpea Intercropping Systems in Eastern Africa. In Silim N. S., Mergeai G., Kimani M. P. (Eds): "Status and Potential of Pigeonpea in Eastern and Southern Africa". Proceedings of a Regional Workshop Nairobi, Kenya, 12-15 Sep 2000, pp 73-83

26. Messager J. L., 1983. Etude la réponse au phosphore de quelques légumineuses fourragères tropicales en régions de savanes centre et nord de Côte d'Ivoire. Mise en place et résultats de première année (1982). $\mathrm{N}^{\circ} 02$ PAT Avril 1983. 32 p.

27. Mulongoy K., Kang B. T., 1986. The role and potential of forages legumes in alley cropping, live mulch and rotation systems in humid and subhumid tropical Africa. Potentials of forages legumes in farming systems of sub-saharan. Proceeding of workshop held at ILCA, Addis-Ababa, Ethiopia 16-19 september 1985, pp 212-231.

28. Ndabalishé I., 1995. Agriculture vivrière Ouest-africaine à travers le cas de la Côte d'Ivoire. Bouaké, Côte d'Ivoire, IDESSA, 383 p. 
29. Ogbe F. M. D., Bamidele J.F., 2007. Potential of Pigeon Pea (Cajanus cajan) for Planted Fallow in Edo State, Nigeria. Asian J. Plant Sei., 6 (3): 490-495

30. Odilon E. C., Asiribo O. E., Ogunlela V. B., Singh B. B., Tarawali S. A., 2007. Strategies to improve and sustain food production capacity in the savanna: The role of leguminous fodder crops in maintaining soil fertility and health. Journal of Food Agriculture \& Environment, Vol. 5, Issue 2, pp 338-344

31. Owino-Gerroh C., Gascho G. J., Phatak S. C., 2005. Pigeonpea Response to Silicon, Phosphorus, and Rhizobium Inoculation in an Acid Coastal Plain Soil. Journal of Plant Nutrition, 28:5, 797-804, DOI: $10.1081 /$ PLN-200055540

32. Rao S. C., Coleman S. W., Mayeux H. S., 2002. Forage Production and Nutritive Value of Selected Pigeon pea Ecotypes in the Southern Great Plains. Crop Science, Vol. 42 No. 4, pp: 1259-1263

33. Schulz S., Honlonkou A. N., Carsky R. J., Manyong V. M.,. Oyewolé B. D., 2003. Alternatives to Mucuna for soil fertility management in southern Bénin: farmer perception and use of traditional and exotic grain legumes. Expl Agric., volume 39, pp. 267-278

34. Sedga Z., 1997. Gestion améliorée de la jachère par l'utilisation des légumineuses de couverture. In Floret C. (ed). "La jachère, lieu de production". CORAF-IRD-UE, Dakar, pp 133-139

35. Shenkute B., Hassen A., Ebro A., Amen N., 2013. Performance of Arsi-Bale kids supplemented with graded levels of pigeonpea in dry season in Mid Rift valley of Ethiopia. Afr. J. Agric. Res. Vol. 8(20), pp. 2366-2370.

36. Skerman P.J.; Peterson R.A.; Riveros F., 1982. Les légumineuses fourragères tropicales. FAO Production Végétale et Protection des Plantes, FAO, $689 \mathrm{p}$

37. Snapp S. S., Rohrbach D.D., Simtowe F., Freeman H.A., 2002. Sustainable soil management options for Malawi: can smallholder farmers grow more legumes? Ecosystems and Environment 91, 159174

38. Snapp S. S., Jones B. R., Minja M. E., Rusiké J., Silim N. S., 2003. Pigeon pea for Africa: A versatile vegetable - And more. Hort Science, Vol. 38 (6), pp 1073-1079

39. Tayo T. O., Togun A. O., 1984. The response of pigeon pea (Cajanus Cajan) to the application of fertilizers. Ann. appl. Biol., 105, 293-302

40. Velay F., Baudoin J.-P., Mergeai G., 2001. Caractérisation du savoir paysan sur les insectes nuisibles du pois d'Angole (Cajanus cajan (L.) Millsp.) dans le Nord de l'Ouganda. Biotechnol. Agron. Soc. Environ. 5 (2), 105-114 
41. Wallis E.S., Faris D.G., Elliott R., Byth D. E., 1986. Varietal improvement of pigeonpea for small-holder livestock production systems. In Workshop on Crop-Livestock Systems Research, July 711, 1986, Khon Kaen, Thailand 